\title{
Ex Vivo Magnetic Sentinel Lymph Node Detection in Colorectal Cancer with a SPIO Tracer
}

\author{
Joost J. Pouw ${ }^{1}$, Raluca M. Fratila ${ }^{1}$, Aldrik H. Velders ${ }^{2}$, Bennie ten Haken ${ }^{1}$, \\ Quentin A. Pankhurst ${ }^{3}$, and Joost M. Klaase ${ }^{4}$ \\ ${ }^{1,2}$ MIRA Institute for Biomedical Technology \& Technical Medicine, \\ Neurolmaging Group $1 /$ Biomedical Chemistry Group ${ }^{2}$, University of Twente \\ P.O. Box 217, 7500AE Enschede, The Netherlands \\ J.J.Pouw@utwente.nl \\ 3 The Royal Institution of Great Britain, Davy-Faraday Research Laboratory \\ 21 Albemarle Street, W1S 4BS, London, UK \\ Q.Pankhurst@endomagnetics.com \\ 4 Medisch Spectrum Twente, Department of Surgery \\ P.O. Box 50.000, 7500 KA Enschede, The Netherlands \\ J.Klaase@mst.nl
}

\begin{abstract}
A new method for selecting sentinel lymph nodes (SNs) in colorectal cancer tissue was investigated in 12 patients. A tracer consisting of superparamagnetic ironoxide (SPIO) nanoparticles was injected in the resected tissue. A handheld magnetic probe was used to select SNs to which the SPIO was drained. Vibrating sample magnetometry was performed on the lymph nodes to quantify the amount of SPIO in the nodes. High-field MRI allowed to depict the distribution of SPIO in the node, and revealed small anatomical structures. One or more SPIO containing nodes were successfully selected with the magnetic probe in all 12 patients.
\end{abstract}

\section{Introduction}

Colorectal cancer is the third most common cancer in men and the second in women, with a total of approximately 1.2 million new cases annually worldwide.[1] In treatment of patients suffering from colorectal cancer and other solid cancers it is very important to determine whether metastatic cells from the primary tumor have migrated to the lymph nodes (LNs). The presence or absence of metastasis in the LNs determines if the patient is treated with chemotherapy or not.

Nodal metastasis can be diagnosed by analyzing a section of surgically resected LNs by light microscopy. Sentinel Lymph Node Mapping (SLNM) is a technique used to select the most relevant LNs. A tracer is injected

T.M. Buzug and J. Borgert (Eds.): Magnetic Particle Imaging, SPPHY 140, pp. 179-183. springerlink.com

(C) Springer-Verlag Berlin Heidelberg 2012 
near the tumor, after which it is drained to the LNs via the lymph vessels that drain the tumor area. The LNs to first receive drainage from the tumor area, are also the nodes first reached by the tracer. Since metastatic cells are drained via the same lymphatic route, these nodes are also most likely to be the first nodes to contain metastasis.[2] The current most commonly used tracer for SLNM in colorectal cancer is a blue dye, which can be visually detected.[3] Recent studies propose to introduce SLNM in colorectal cancer to identify very small metastasis in the SNs which are missed during regular light microscopic analysis. $[4,5]$

We have used a SPIO tracer and a commercially available handheld magnetic detection device (SentiMag®, Endomagnetics LTD., London, UK) to select the Sentinel Nodes (SNs) in colorectal cancer tissue. The SentiMag $®$ is originally developed to intraoperatively detect SNs in breast cancer.[6]

\section{Material and Methods}

In surgical treatment of colorectal cancer the tumor and complete adjacent draining lymphatic tissue is resected in one piece. This allows to perform SLNM after resection, ex vivo. When the tracer is injected after resection the patient is not exposed to the substance, and there is no chance of adverse events. Because the patient is not exposed, also newly developed substances not (yet) approved for use in humans can be tested. Ex vivo SLNM can be performed as effective as in vivo in colorectal cancer.[7]

We analysed the distribution of a SPIO tracer consisting of nanoparticles, approved for use in humans (Endorem®, Guerbet Nederland BV, Gorinchem, The Netherlands; diameter: $80-150 \mathrm{~nm}, 11.2 \mathrm{mg} \mathrm{Fe} \mathrm{mL}^{-1}$ ), to the LNs in colorectal tissue. We aimed to select the SNs with the SPIO tracer and SentiMag $\circledast$ handheld probe.

12 patients undergoing surgical resection for colorectal cancer in the MST Enschede hospital gave informed consent. Immediately following resection of the colorectal segment, approximately $2 \mathrm{~mL}$ of Endorem was injected submucosally near the tumor. A gentle massage of the injection site was performed to promote lymphatic transport of the tracer. A blue dye (Patent Blue V, Guerbet Nederland BV, Gorinchem, The Netherlands) was administered as a reference, in a similar fashion, because this is currently the most used method in colorectal SLNM studies.

After resection of all the LNs from the tissue by the pathologist, the SentiMag $\AA$ was used to select the lymph nodes containing SPIO. The nodes were placed on the probe, and the measured signal intensity was recorded for each node (Fig. 1). 


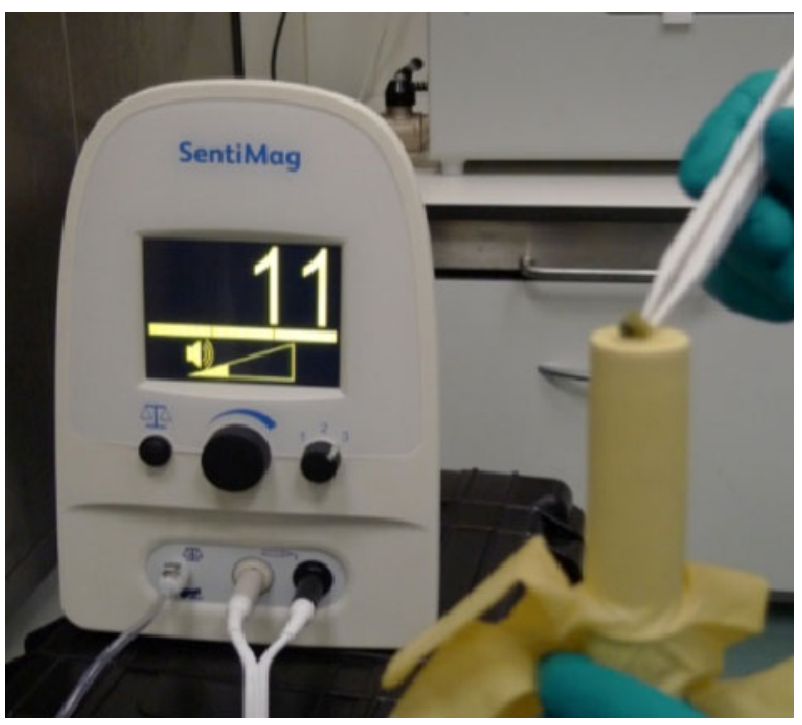

Fig. 1. SentiMag® control unit and handheld probe (covered by surgical glove to prevent contamination) with LN placed on the probe to asses the presence of SPIO in the node.

Blue discoloration of the LNs due to the presence of Patent Blue $\mathrm{V}$ was visually detected and noted.

In a subset of the patients, all SPIO containing LNs were subjected to a Vibrating Sample Magnetometry (VSM) measurement to accurately quantify the amount of iron present.

High-field (14.1 T, Bruker Avance II NMR spectrometer) T1- and T2weighted MR imaging was carried out in order to assess the distribution of SPIO inside the individual LNs.

As a final step, the LNs were subjected to histopathological examination to determine whether metastasis was present, and iron specific staining with Perls Prussian Blue to microscopically investigate the iron distribution in the nodes.

\section{Results}

The SPIO tracer was successfully transported through the lymphatic system into the SNs in all patients. One or more SPIO containing LNs were selected in each of the 12 patients using the SentiMag®. The amount of iron in the LNs measured by VSM ranged between 2-142 $\mu \mathrm{g}$. The measured amount of iron by VSM was linearly related to the signal intensity displayed by the SentiMag $\AA$, which indicates that the SentiMag $\AA$ can be used as an indication of the amount of iron present in the lymph nodes. 
High-field MR images of some of the nodes showed hypointense areas due to the presence of SPIO. Small anatomical structures were also revealed on these images. An example in which both the hypointense areas and the small anatomical structures (follicles) can be distinguished can be seen in Fig. 2.

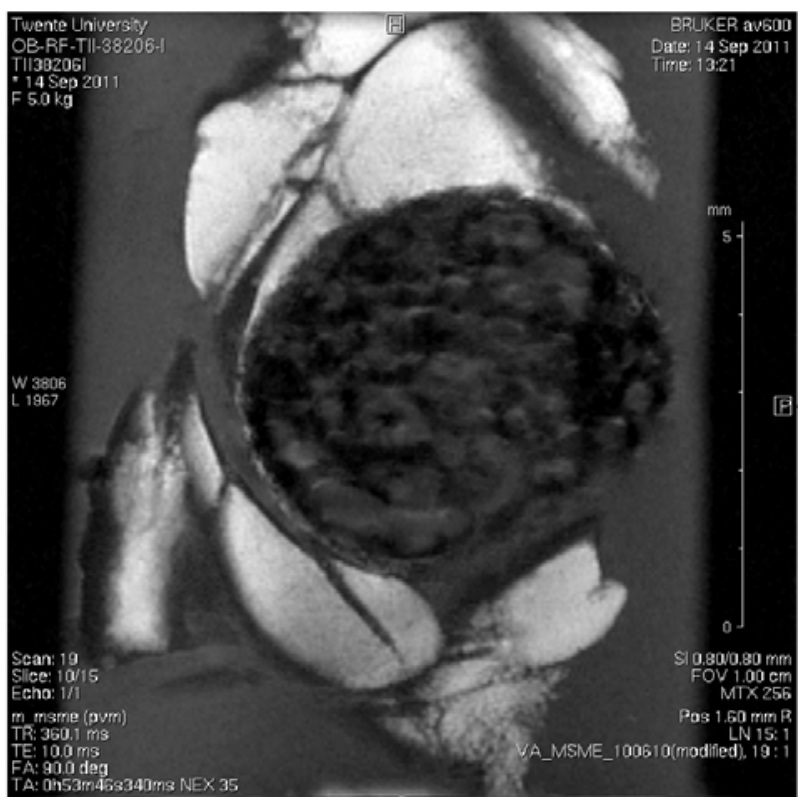

Fig. 2. T1-weighted image of a $S N$, surrounded by fat tissue. The image was acquired using a multi-slice-multi-echo (MSME) sequence.

\section{Discussion and Conclusion}

These initial experiment showed that it is possible to select SNs in colorectal tissue with the SentiMag® after ex vivo administration of a SPIO tracer. To validate the clinical applicability of this method in colorectal cancer, it is necessary to correlate the presence of metastasis in the LNs to the presence and/or amount of iron found in nodes. We are currently performing this analysis on the cohort of 12 patients, and including a larger number of patients to be able to draw firm conclusions.

Acknowledgments. This work was supported by the Dutch Technology Foundation STW under Project Radiation free localization and diagnosis of sentinel lymph nodes with magnetic nano-particles. 


\section{References}

1. International Agency for Research on Cancer, GLOBOSCAN (2008), http: //globoscan.iarc. fr

2. Tan, K.Y., et al.: Distribution of the first metastatic lymph node in colon cancer and its clinical significance. Colorectal Dis. 12(1), 44-47 (2010)

3. Cahill, R.A., Leroy, J., Marescaux, J.: Could lymphatic mapping and sentinel node biopsy provide oncological providence for local resectional techniques for colon cancer? A review of the literature. BMC Surg. 8, 17 (2008)

4. van der Zaag, E.S., et al.: Diagnosing occult tumour cells and their predictive value in sentinel nodes of histologically negative patients with colorectal cancer. Eur. J. Surg. Oncol. 36(4), 350-357 (2010)

5. van Schaik, P.M., et al.: Ex vivo sentinel lymph node "mapping" in colorectal cancer. Eur. J. Surg. Oncol. 33(10), 1177-1182 (2007)

6. Joshi, T., et al.: Magnetic nanoparticles for detecting sentinel lymph nodes. ESJO 33, 1135 (2007)

7. Park, J.S., et al.: Comparison of ex vivo and in vivo injection of blue dye in sentinel lymph node mapping for colorectal cancer. World J. Surg. 33(3), 539-546 (2009) 